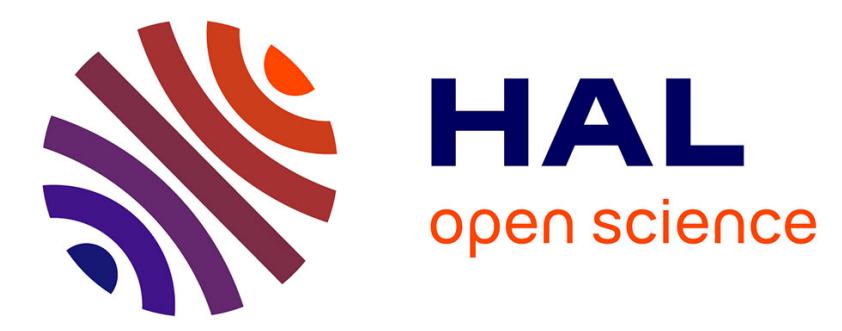

\title{
Exemple de résolution numérique de l'équation caractéristique d'une cavité hyperfréquence contenant un échantillon diélectrique
}

\author{
A. Poinsot
}

\section{- To cite this version:}

A. Poinsot. Exemple de résolution numérique de l'équation caractéristique d'une cavité hyperfréquence contenant un échantillon diélectrique. Revue de Physique Appliquée, 1977, 12 (7), pp.1055-1062. 10.1051/rphysap:019770012070105500 。 jpa-00244277

\section{HAL Id: jpa-00244277 https://hal.science/jpa-00244277}

Submitted on 1 Jan 1977

HAL is a multi-disciplinary open access archive for the deposit and dissemination of scientific research documents, whether they are published or not. The documents may come from teaching and research institutions in France or abroad, or from public or private research centers.
L'archive ouverte pluridisciplinaire HAL, est destinée au dépôt et à la diffusion de documents scientifiques de niveau recherche, publiés ou non, émanant des établissements d'enseignement et de recherche français ou étrangers, des laboratoires publics ou privés. 


\title{
EXEMPLE DE RÉSOLUTION NUMÉRIQUE DE L'ÉQUATION CARACTÉRISTIQUE D'UNE CAVITÉ HYPERFRÉQUENCE CONTENANT UN ÉCHANTILLON DIÉLECTRIQUE
}

\author{
A. POINSOT \\ Laboratoire de Spectronomie $(*)$, groupe de Radioélectricité, \\ Université de Dijon, 6 Bd Gabriel 21000 Dijon, France.
}

(Reçu le 17 janvier 1977, accepté le 6 avril 1977)

\begin{abstract}
Résumé. - La résonance d'une cavité hyperfréquence contenant un échantillon diélectrique, peut être décrite par une équation caractéristique. Nous avons exploré quelques solutions numériques de cette équation, dans le cas des modes $\mathrm{TM}_{1 \text { mo }}$ de cavités cylindriques qui sont souvent utilisés pour la caractérisation des paramètres diélectriques de l'échantillon. Lorsque le domaine de variation de ces paramètres est étendu, on voit apparaître deux comportements différents de la résonance, l'un caractéristique d'un échantillon à faibles pertes, l'autre caractéristique d'un échantillon à fortes pertes. Ces deux comportements peuvent être mis en évidence, expérimentalement, avec un échantillon ferroélectrique dont la permittivité complexe présente d'importantes variations avec la température.
\end{abstract}

\begin{abstract}
The resonance of a microwave cavity containing a dielectric sample can be described by a characteristic equation. We have investigated some of the numerical solutions of this equation, for the case of TM 1 mo modes for cylindrical cavities, which are often used for the characterization of the dielectric parameters of the sample. When the domain of variation of these parameters is large, two different comportments of the resonance emerge, the first being characteristic of a sample with small losses, the second, characteristic of a sample with high losses. These two comportments can be demonstrated, experimentally, with a ferroelectric sample for which the complex permittivity shows large variations with temperature.
\end{abstract}

1. Introduction. - Le problème de la cavité hyperfréquence contenant un échantillon diélectrique est susceptible d'une solution approchée, largement utilisée, sous la forme d'une formule des perturbations.

La méthode plus rigoureuse, qui consiste à résoudre l'équation caractéristique, conduit le plus souvent à des calculs qui ne peuvent être résolus que numériquement à l'aide de moyens de calculs assez importants. Toutefois la dissémination croissante de ces moyens de calcul, tend à favoriser la méthode de résolution de l'équation caractéristique au détriment de la méthode des perturbations.

Nous présentons ici quelques exemples de résultats obtenus par résolution de l'équation caractéristique, qui présentent la particularité de s'écarter nettement du domaine d'application de la théorie des perturbations.

2. Etude théorique des modes $\mathbf{T} \mathbf{M}_{\text {omo }}$ - - La figure 1 présente la géométrie du modèle étudié. On peut remarquer que la hauteur $L$ de la cavité n'interviendra pas dans les propriétés des modes étudiés ici.

(*) (E. R. A. n० 599).

REVUE DE PHYSIQUE APPLIQUÉE. - T. 12, N• 7, JUILLET 1977
Dans l'hypothèse simplificatrice d'une cavité parfaitement conductrice et très faiblement couplée l'équation caractéristique des modes $\mathrm{TM}_{\text {omo }}$ s'écrit [1] :

$$
\left|\begin{array}{ccc}
-J_{0}\left(R_{1} k_{1}\right) & J_{0}\left(R_{1} k_{2}\right) & Y_{0}\left(R_{1} k_{2}\right) \\
0 & J_{0}\left(R_{2} k_{2}\right) & Y_{0}\left(R_{2} k_{2}\right) \\
-k_{1} J_{1}\left(R_{1} k_{1}\right) & k_{2} J_{1}\left(R_{1} k_{2}\right) & k_{2} Y_{1}\left(R_{1} k_{2}\right)
\end{array}\right|=0
$$

Il y a lieu d'interpréter cette équation en valeurs complexes. La permittivité relative de l'échantillon diélectrique s'écrira donc

$$
\varepsilon_{\mathrm{r} 1}=\varepsilon_{\mathrm{r}}^{\prime}-j \varepsilon_{\mathrm{r}}^{\prime \prime}=\varepsilon_{\mathrm{r}}^{\prime}(1-\mathrm{jtg} \delta),
$$

et pour l'air emplissant le reste de la cavité on a supposé $\varepsilon_{\mathrm{r} 2}=1$.

Nous écrirons la fréquence complexe sous la forme

$$
\vec{v}=v(1+j \alpha)
$$

où $v$ est la fréquence centrale du mode de résonance et $\alpha=1 / 2 Q$ est le facteur d'amortissement qui traduit l'influence des pertes diélectriques sur la largeur du mode de résonance [2]. La largeur mesurée du mode de résonance inclu également les pertes dans les parois 


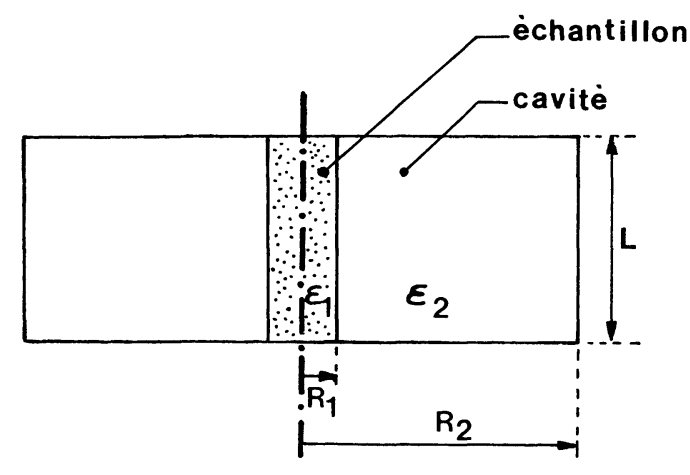

Fig. 1. - Géométrie du modèle étudié.

[Geometry of the studied model.]

et par les couplages. On devra donc, pour déterminer $\alpha$ expérimentalement, utiliser la relation

$$
\alpha=\frac{1}{2 Q_{\text {total }}}-\left(\frac{1}{2 Q_{\text {parois }}}+\frac{1}{2 Q_{\text {couplage }}}\right) .
$$

Pour terminer l'interprétation de l'équation caractéristique on posera

$$
k_{1}=\frac{2 \pi \bar{v}}{c} \sqrt{\varepsilon_{\mathrm{r} 1}} \text { et } \quad k_{2}=\frac{2 \pi \bar{v}}{c} .
$$

Les fonctions de Bessel de $1^{\text {re }}$ et $2^{\mathrm{e}}$ espèce qui figurent dans l'équation caractéristique sont donc d'arguments complexes. En séparant partie réelle et partie imaginaire de l'équation (1), on est conduits à la résolution d'un système de 2 équations non linéaires. Nous avons mis au point des programmes qui permettent le calcul de

$$
v=f\left(\varepsilon_{\mathrm{r}}^{\prime}, \varepsilon_{\mathrm{r}}^{\prime \prime}\right) \quad \text { et } \quad \alpha=g\left(\varepsilon_{\mathrm{r}}^{\prime}, \varepsilon_{\mathrm{r}}^{\prime \prime}\right)
$$

et le calcul inverse de

$$
\varepsilon_{\mathrm{r}}^{\prime}=h(v, \alpha) \quad \text { et } \quad \varepsilon_{\mathrm{r}}^{\prime \prime}=t(v, \alpha),
$$

les dimensions géométriques étant données.

On remarque que l'indice radial $m$ du mode n'intervient pas dans l'équation (1). En fait la résolution de (1) fournit toujours une suite infinie de solutions qui correspondent aux valeurs successives de l'indice $m$. On dira, en ce sens, que les modes $\mathrm{TM}_{\text {omo }}$ forment une famille.

Nous présentons maintenant quelques résultats particuliers obtenus par l'exploitation de nos programmes. Ces résultats concernent une cavité de rayon $R_{2}=19,98 \mathrm{~mm}$, contenant un échantillon de rayon $R_{1}=1 \mathrm{~mm}$. Nous avons étudié, à géométrie constante, la variation des paramètres $v$ et $\alpha$ de la résonance en fonction des paramètres $\varepsilon_{\mathrm{r}}^{\prime}$ et $\varepsilon_{\mathrm{r}}^{\prime \prime}$ de l'échantillon.

La figure 2 montre l'évolution des paramètres $v$ et $\alpha$ de la cavité (modes $\mathrm{TM}_{010}$ et $\mathrm{TM}_{020}$ ) en fonction de la partie réelle $\varepsilon_{\mathrm{r}}^{\prime}$ de la permittivité de l'échantillon pour un facteur de pertes constant $\operatorname{tg} \delta=0,1$.
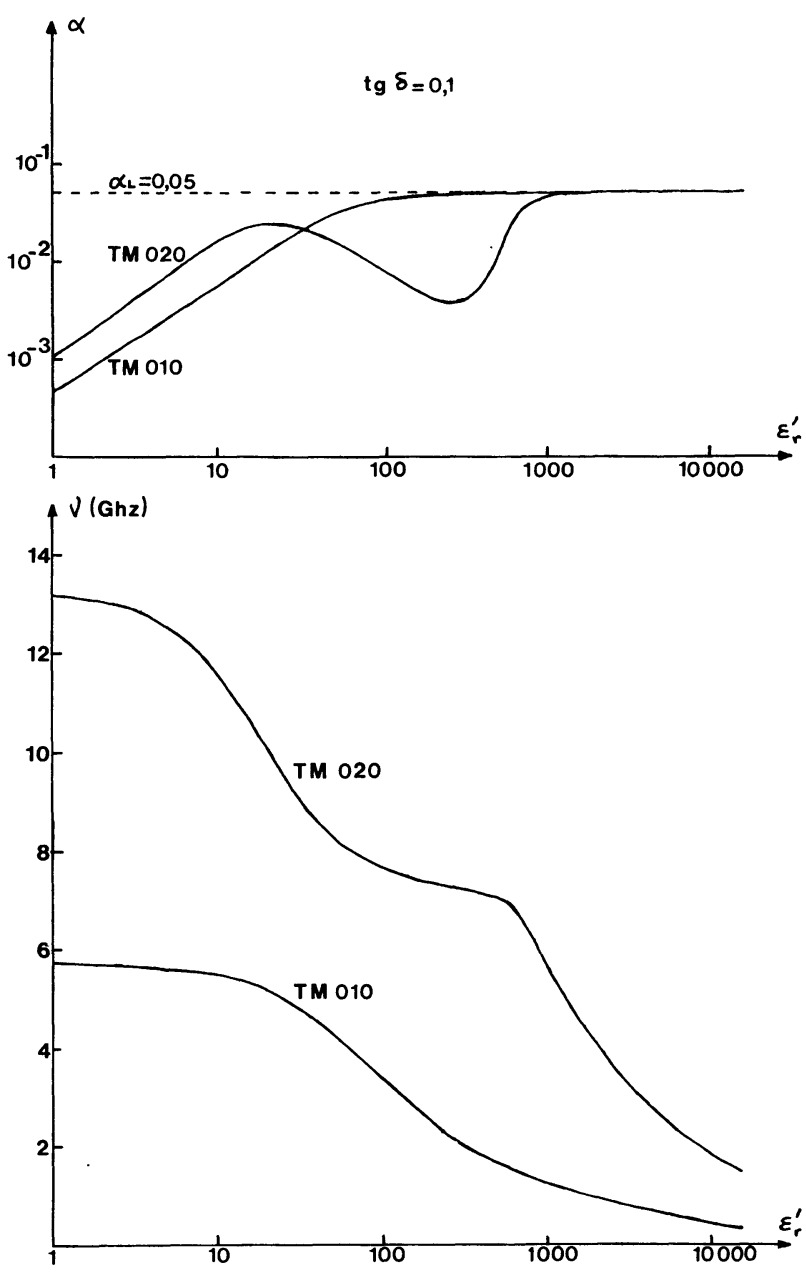

Fig. 2. - Fréquence de résonance et coefficient d'amortissement des modes $\mathbf{T M}_{010}$ et $\mathbf{T M}_{020}$ dans une cavité $\left(R_{2}=19,98 \mathrm{~mm}\right)$ contenant un échantillon diélectrique $\left(R_{1}=1 \mathrm{~mm}\right)$ de facteur de pertes $\operatorname{tg} \delta=0,1$.

[Resonant frequency and damping coefficient for $\mathrm{TM}_{010}$ and $\mathrm{TM}_{020}$ modes, in a cavity $\left(R_{2}=19.98 \mathrm{~mm}\right)$ containing a dielectric sample $\left(R_{1}=1 \mathrm{~mm}\right)$ with loss factor $\operatorname{tg} \delta=0.1$.]

Ces courbes appellent plusieurs remarques. D'abord, la théorie des perturbations, au premier ordre, donne seulement la pente à l'origine des courbes présentées ici, ce qui permet de se rendre compte du domaine de validité de cette théorie.

On remarque ensuite sur le diagramme $v=f\left(\varepsilon_{\mathrm{r}}^{\prime}\right)$ une variation en marche d'escaliers qui serait encore plus sensible pour les modes supérieurs de la famille que nous n'avons pas représentés. Ce phénomène est très général et s'observe pour toutes les géométries de cavités [3]. Il traduit l'accumulation dans l'échantillon, des différents ventres de champ électrique stationnaire. Ainsi le mode $\mathrm{TM}_{010}$ qui ne possède, radialement, qu'un ventre de champ électrique présente une seule marche, le mode $\mathrm{TM}_{020}$ présente deux marches, et ainsi de suite. Les variations de fréquence sont stationnaires lorsque la frontière de l'échantillon se trouve au niveau d'un nœud de champ électrique tangentiel, et rapides lorsque la frontière de l'échantillon se trouve au voisinage d'un ventre de champ électrique tangentiel. 
Les courbes $\alpha=g\left(\varepsilon_{\mathrm{r}}^{\prime}\right)$ à tg $\delta$ donné montrent corrélativement que l'amortissement passe par un maximum lorsque la fréquence de résonance varie rapidement, et par un minimum lorsqu'elle est stationnaire. Lorsque tous les ventres du champ électrique stationnaire sont concentrés dans l'échantillon, l'amortissement tend vers la valeur limite $\alpha_{\mathrm{L}}=\frac{1}{2} \operatorname{tg} \delta$, et tout se passe alors, du point de vue de l'amortissement, comme si l'échantillon remplissait entièrement la cavité.

La figure 3 montre, pour les mêmes modes $\mathrm{TM}_{010}$ et $\mathrm{TM}_{\mathbf{0 2 0}}$, l'évolution des paramètres de la cavité $v=f\left(\varepsilon_{\mathrm{r}}^{\prime}\right)$ et $\alpha=g\left(\varepsilon_{\mathrm{r}}^{\prime}\right)$ dans le cas d'un échantillon présentant un facteur de pertes $\operatorname{tg} \delta=0,5$.

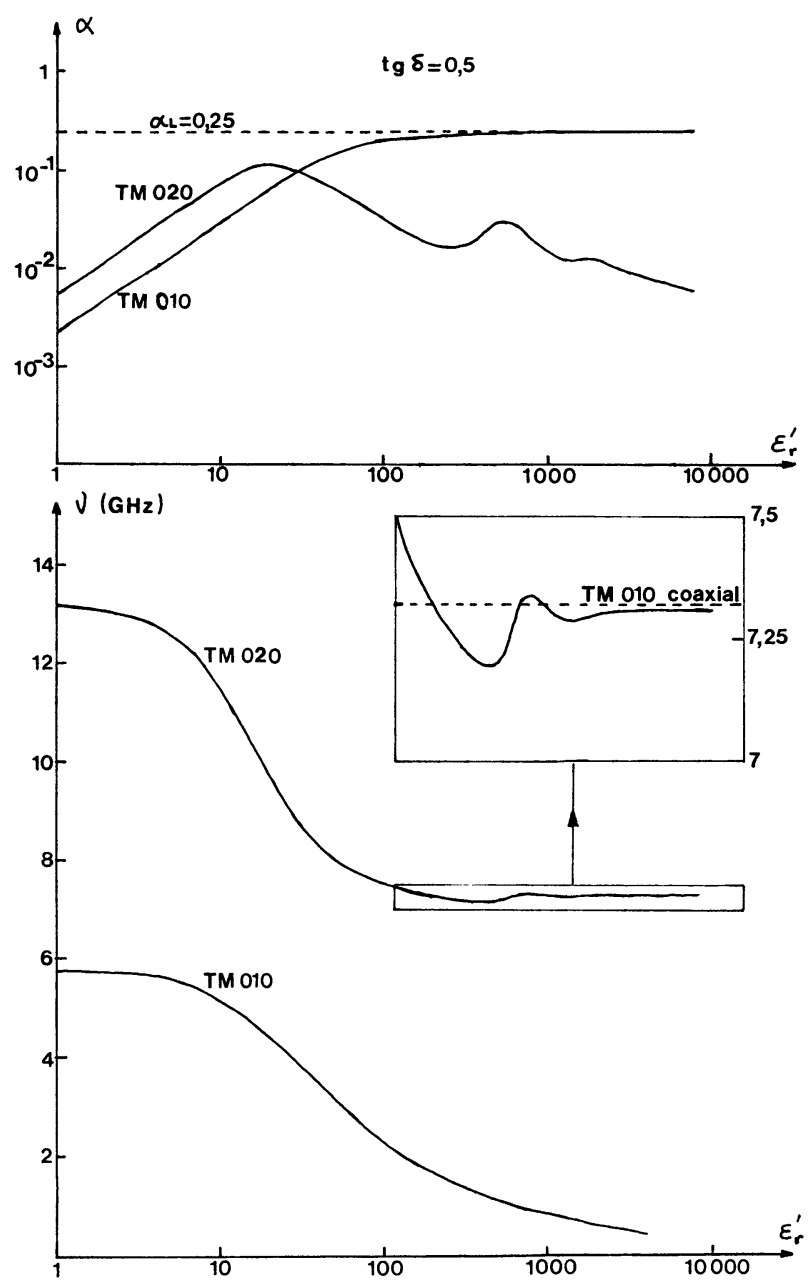

FIG. 3. - Fréquence de résonance et coefficient d'amortissement des modes $\mathrm{TM}_{010}$ et $\mathrm{TM}_{020}$ dans une cavité $\left(R_{2}=19,98 \mathrm{~mm}\right)$ contenant un échantillon diélectrique $\left(R_{1}=1 \mathrm{~mm}\right)$ de facteur de pertes $\operatorname{tg} \delta=0,5$.

[Resonant frequency and damping coefficient for $\mathrm{TM}_{010}$ and $\mathrm{TM}_{020}$ modes, in a cavity $\left(R_{2}=19.98 \mathrm{~mm}\right)$ containing a dielectric sample $\left(R_{1}=1 \mathrm{~mm}\right)$ with loss factor $\operatorname{tg} \delta=0.5$.]

Pour le mode $\mathrm{TM}_{010}$ les courbes sont analogues à celles de la figure 2 si ce n'est une translation de l'amortissement vers des valeurs plus élevées. Par contre le mode $\mathrm{TM}_{020}$ (et tous les modes supérieurs de la famille) présente un comportement nouveau. L'accu- mulation des ventres de champ électrique stationnaire dans l'échantillon se limite au seul ventre central. La fréquence de résonance reste ensuite stationnaire (avec quelques oscillations de faible amplitude). Elle tend vers une valeur limite qui est la fréquence de résonance $\mathrm{du}$ mode $\mathrm{TM}_{010}$ coaxial que l'on observerait dans la cavité en métallisant la surface de l'échantillon. On peut dire, dans cette région, que l'échantillon diélectrique présente plutôt un comportement métallique. On voit aussi que l'amortissement $\alpha$ de la cavité reste toujours inférieur à la valeur limite $\alpha_{\mathrm{L}}$ et qu'il diminue nettement lorsque l'échantillon présente son comportement métallique.

$\mathrm{La}$ résolution, en valeurs complexes de l'équation caractéristique (1) met donc en évidence deux comportements très différents de la fréquence de résonance et de l'amortissement d'une cavité contenant un échantillon diélectrique, selon la valeur du facteur de pertes $\operatorname{tg} \delta$ de cet échantillon. Nous allons retrouver un phénomène analogue pour la familles des modes $\mathrm{TM}_{\mathbf{l m o}}$.

3. Etude théorique des modes $\mathbf{T} \mathbf{M}_{\mathrm{Imo}} \cdot$ - L'équation caractéristique des modes $\mathrm{TM}_{\mathrm{lmo}}$ s'écrit :

$$
\left|\begin{array}{ccc}
-J_{1}\left(R_{1} k_{1}\right) & J_{1}\left(R_{1} k_{2}\right) & \left.Y_{1} R_{1} k_{2}\right) \\
0 & J_{1}\left(R_{2} k_{2}\right) & Y_{1}\left(R_{2} k_{2}\right) \\
-k_{1} J_{1}^{\prime}\left(R_{1} k_{1}\right) & k_{2} J_{1}^{\prime}\left(R_{1} k_{2}\right) & k_{2} Y_{1}^{\prime}\left(R_{1} k_{2}\right)
\end{array}\right|=0
$$

Cette équation fait intervenir les fonctions de Bessel, d'ordre 1 , de première et seconde espèce, et leurs dérivées.

Nous étudions ici seulement le premier mode $\left(\mathrm{TM}_{110}\right)$ de la famille $\mathrm{TM}_{\mathrm{lmo}}$. En effet des études complémentaires nous ont montré que tous les modes de cette famille présentent les mêmes propriétés.

La figure 4 permet de comparer les résultats dans un cas à faibles pertes $(\operatorname{tg} \delta=0,1)$ et dans un cas à fortcs pertes $(\operatorname{tg} \delta=0,5)$.

Dans le cas où $\operatorname{tg} \delta=0,1$ l'échantillon présente un comportement diélectrique. Lorsque $\varepsilon_{\mathrm{r}}^{\prime}$ croît la fréquence reste d'abord stationnaire tandis que l'amortissement augmente régulièrement. Ceci peut être relié au fait que la frontière de l'échantillon reste proche du nœud central de champ électrique. Lorsque le ventre de champ électrique, qui se déplace vers le centre de la cavité, atteint la frontière de l'échantillon la fréquence décroît rapidement, tandis que l'amortissement présente une saturation à la valeur $\alpha_{\mathrm{L}}=\frac{1}{2} \operatorname{tg} \delta$. Le champ électrique est alors localisé dans l'échantillon.

Pour $\operatorname{tg} \delta=0,5$ l'échantillon manifeste au contraire son comportement métallique. On observe une stabilisation de la fréquence de résonance qui tend, après quelques oscillations, vers la fréquence du mode $\mathrm{TM}_{110}$ coaxial qui résonnerait dans la cavité après métallisation de la surface de l'échantillon. La figure 5 présente un agrandissement de la courbe $v=f\left(\varepsilon_{\mathrm{r}}^{\prime}\right)$, où l'on observe clairement la différence des comportements. Le comportement diélectrique s'accompagne 


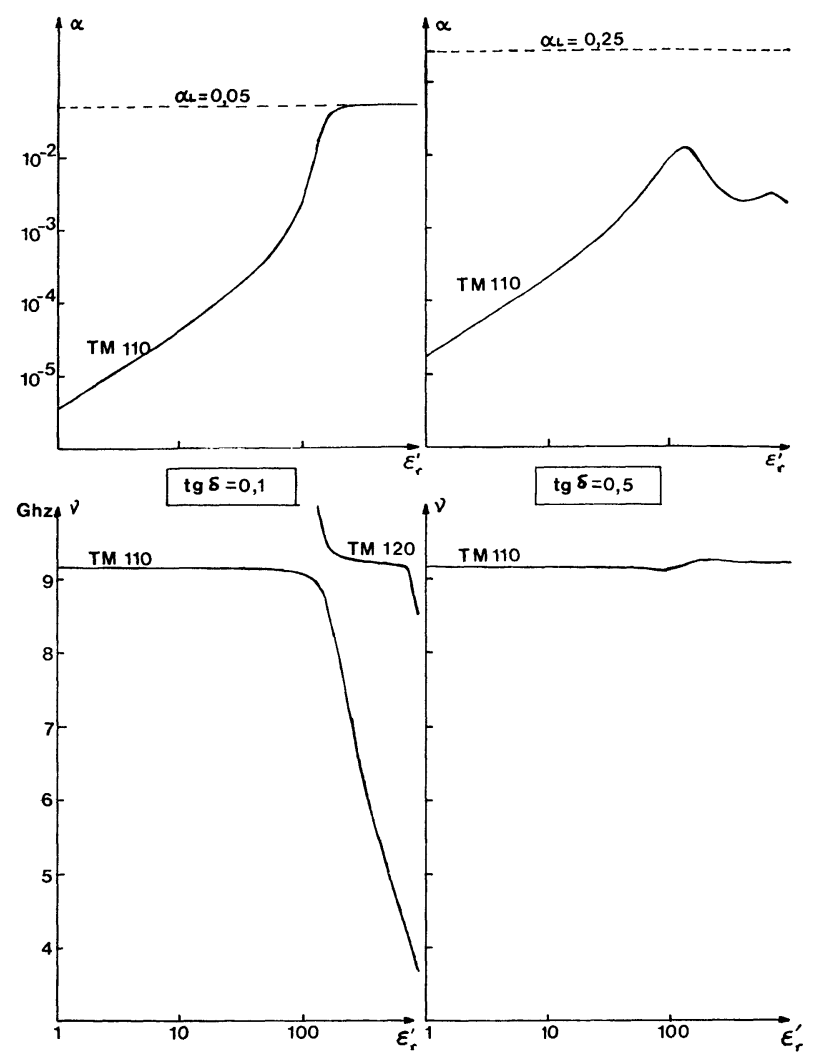

Fig. 4. - Fréquence de résonance et coefficient d'amortissement du mode $\mathrm{TM}_{110}$ dans une cavité $\left(R_{2}=19,98 \mathrm{~mm}\right)$ contenant un échantillon diélectrique $\left(R_{1}=1 \mathrm{~mm}\right)$ de facteur de pertes $\operatorname{tg} \delta$. Comparaison des comportements pour tg $\delta=0,1$ et $\operatorname{tg} \delta=0,5$.

[Resonant frequency and damping coefficient for $\mathrm{TM}_{110}$ mode, in a cavity $\left(R_{2}=19.98 \mathrm{~mm}\right)$ containing a dielectric sample $\left(R_{1}=1 \mathrm{~mm}\right)$ with loss factor $\operatorname{tg} \delta$. Comparison of comportments for $\operatorname{tg} \delta=0.1$ and $\operatorname{tg} \delta=0.5$.]

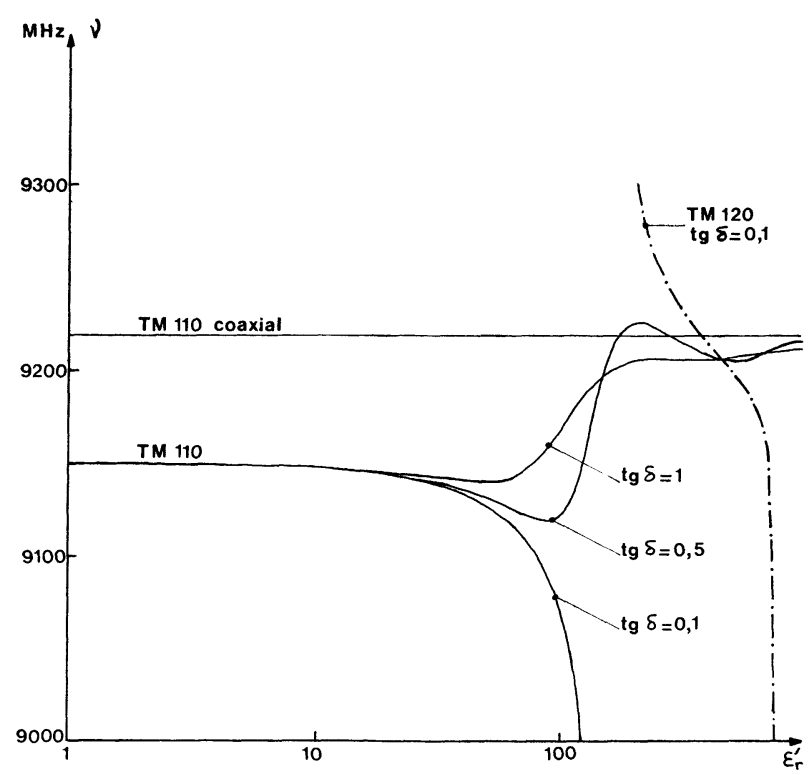

Fig. 5. - Agrandissement des courbes de fréquences de la figure 4 .

[Magnification of the frequency curves of the figure 4.] d'une chute brutale de la fréquence de résonance, le comportement métallique se traduit par une remontée de la fréquence de résonance au-dessus du niveau de la cavité vide.

Cette transition diélectrique-métal ne correspond évidemment à aucune transformation physique de l'échantillon. Ce n'est qu'une terminologie utilisée pour décrire deux comportements macroscopiques très différents de l'échantillon, vis-à-vis de la pénétration des ondes électromagnétiques. Il faut remarquer que cette transition du comportement se produit à l'intérieur d'un domaine très limité de $\operatorname{tg} \delta$ (ici entre $\operatorname{tg} \delta=0,1$ et $\operatorname{tg} \delta=0,5$ ).

On remarque encore sur la figure 4 que l'amortissement $\alpha$ reste toujours très inférieur à la valeur de saturation $\alpha_{L}=0,25$, dans le cas où $\operatorname{tg} \delta=0,5$, et qu'il diminue lorsque l'échantillon présente un comportement métallique, si bien qu'un échantillon de facteur de pertes $\operatorname{tg} \delta=0,5$ donne alors une résonance plus aiguë qu'un échantillon de facteur de pertes $\operatorname{tg} \delta=0,1$.

Nous avons tenté, sur les figures 6 et 7, de résumer l'ensemble des phénomènes, par une représentation tridimensionnelle des surfaces caractéristiques

$$
v=f\left(\varepsilon_{\mathrm{r}}^{\prime}, \operatorname{tg} \delta\right) \quad \text { et } \quad \alpha=g\left(\varepsilon_{\mathrm{r}}^{\prime}, \operatorname{tg} \delta\right)
$$

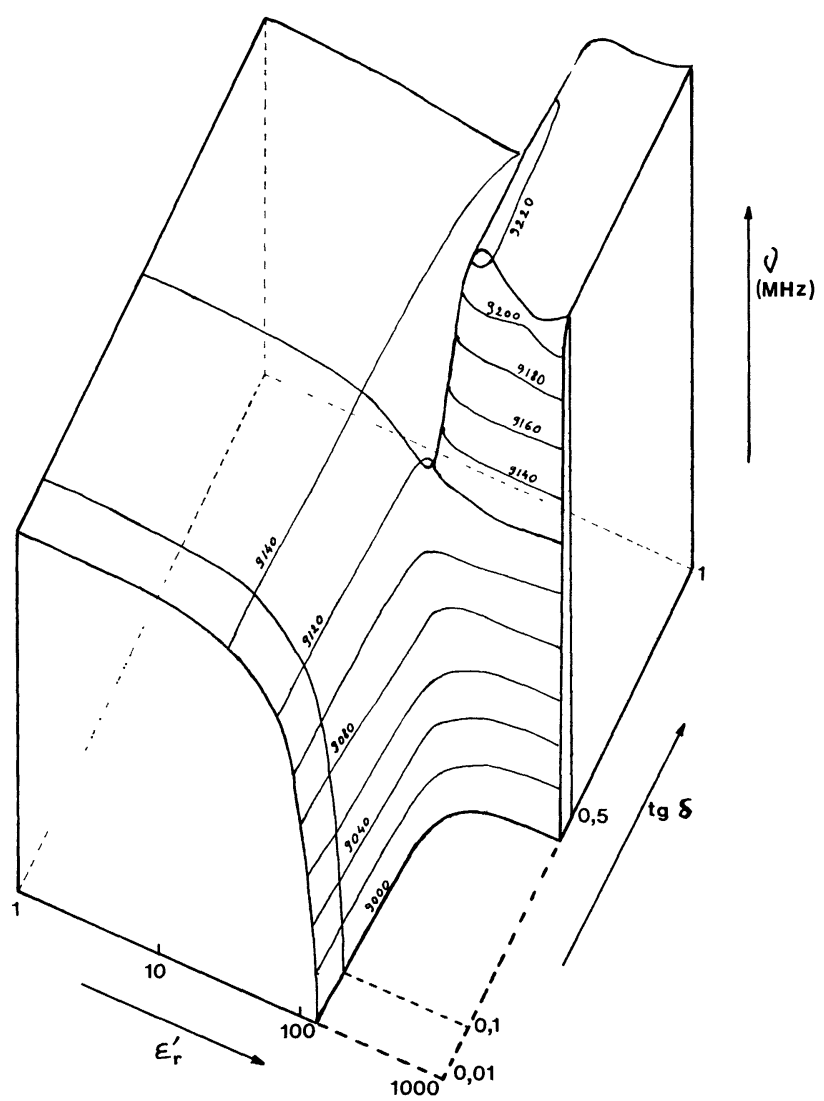

Fig. 6. - Représentation à 3 dimensions de la surface caractéristique $v=f\left(\varepsilon_{\mathrm{r}}^{\prime}, \operatorname{tg} \delta\right)$ pour le mode $\mathrm{TM}_{110}$ dans une cavité $\left(R_{2}=19,98 \mathrm{~mm}\right)$ contenant un échantillon diélectrique $\left(R_{1}=1 \mathrm{~mm}\right)$ de paramètres $\varepsilon_{\mathrm{r}}^{\prime}, \operatorname{tg} \delta$.

[Three dimensional view of the characteristic surface $v=f\left(\varepsilon_{\mathrm{r}}^{\prime}, \operatorname{tg} \delta\right)$ for the mode $\mathrm{TM}_{110}$ in a cavity $\left(R_{2}=19.98 \mathrm{~mm}\right)$ containing a dielectric sample $\left(R_{1}=1 \mathrm{~mm}\right)$ with parameters $\varepsilon_{r}^{\prime}, \operatorname{tg} \delta$.] 


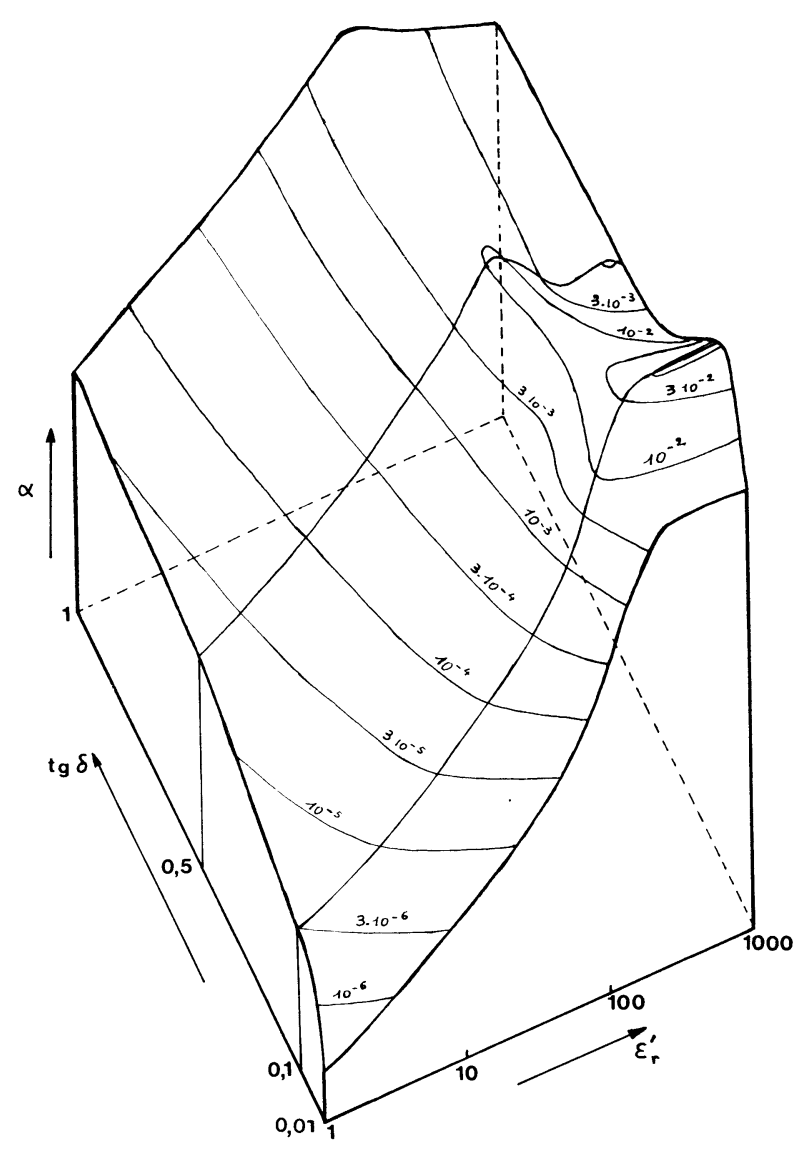

FIG. 7. - Représentation à 3 dimensions de la surface caractéristique $\alpha=g\left(\varepsilon_{\mathrm{r}}^{\prime}, \operatorname{tg} \delta\right)$ pour le mode $\mathrm{TM}_{110}$ dans une cavité $\left(R_{2}=19,98 \mathrm{~mm}\right)$ contenant un échantillon diélectrique $\left(R_{1}=1 \mathrm{~mm}\right)$ de paramètres $\varepsilon_{\mathrm{r}}^{\prime}, \operatorname{tg} \delta$.

[Three dimensional view of the characteristic surface $\alpha=f\left(\varepsilon_{\mathrm{r}}^{\prime}\right.$, $\operatorname{tg} \delta$ ) for the mode $\mathrm{TM}_{110}$ in a cavity $\left(R_{2}=19.98 \mathrm{~mm}\right)$ containing a dielectric sample $\left(R_{1}=1 \mathrm{~mm}\right)$ with parameters $\varepsilon_{\mathrm{r}}^{\prime}, \operatorname{tg} \delta$.]

On voit en particulier sur la figure 7 que l'amortissement maximum ne coïncide pas avec les valeurs les plus élevées de $\operatorname{tg} \delta$.

4. Etude expérimentale d'une cavité contenant un échantillon de TGS. - Nous avons réalisé une étude expérimentale avec le mode $\mathrm{TM}_{120}$. Il permet en effet d'obtenir, pour une fréquence donnée, des dimensions de cavité plus grandes que le mode $\mathrm{TM}_{110}$, ce qui facilite la réalisation. Il s'agit d'un mode avec nœud central de champ électrique, qui possède des propriétés analogues à celles du mode $T M_{110}$. Dans la famille des modes $\mathrm{TM}_{1 \mathrm{mo}}$, il n'y a pas lieu de distinguer le premier mode de la famille et les modes supérieurs, comme c'était le cas dans la famille des modes $\mathrm{TM}_{\mathrm{omo}}$.

Pour l'échantillon notre choix s'est porté sur le ferroélectrique Tri-glycine Sulfate (T. G. S.) de formule $\left(\mathrm{CH}_{2} \mathrm{NH}_{2} \mathrm{COOH}\right)_{3} \cdot \mathrm{H}_{2} \mathrm{SO}_{4}$. Il est possible de tailler dans un monocristal un échantillon cylindrique tel que l'axe du cylindre coïncide avec l'axe ferroélectrique du cristal. Le champ électrique du mode $\mathrm{TM}_{120}$ étant purement axial, seule la permittivité selon l'axe ferroélectrique interviendra. Cette permittivité présente, en fonction de la température, des variations très importantes, comme le montrent les courbes 1 et 2 de la figure 8 [4], [5]. Il est ainsi possible, avec un seul échantillon, mais en faisant varier la température dans une plage facilement accessible de $25^{\circ} \mathrm{C}$ à $100^{\circ} \mathrm{C}$, d'étudier le comportement de la cavité pour différentes valeurs de la permittivité complexe de l'échantillon.
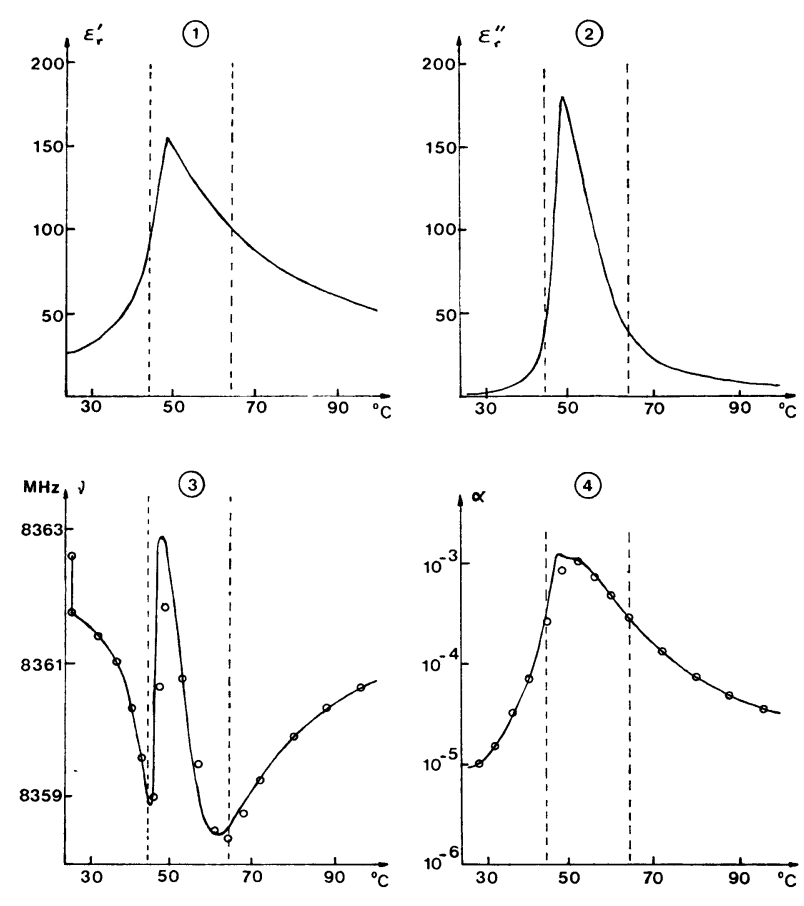

0 : points experimentaux

Fig. 8. - Etude, en fonction de la température d'une cavité $\left(R_{2}=40 \mathrm{~mm}\right)$ résonnant sur le mode $\mathrm{TM}_{120}$ et contenant un échantillon de TGS $\left(R_{1}=0,73 \mathrm{~mm}\right) 1$ et $2:$ Variations avec la température des paramètres $\varepsilon_{\mathrm{r}}^{\prime}$ et $\varepsilon_{\mathrm{r}}^{\prime \prime}$ de TGS 3 et 4 : Variations correspondantes des paramètres $v$ et $\alpha$ de la cavité. Comparaison entre les valeurs calculées et les valeurs expérimentales.

[Study, with temperature, of a cavity $\left(R_{2}=40 \mathrm{~mm}\right)$ resonating on the mode $\mathrm{TM}_{120}$ and containing a sample of TGS $\left(R_{1}=0.73 \mathrm{~mm}\right) .1$ et $2:$ Variations, with temperature, of the parameters $\varepsilon_{\mathrm{r}}^{\prime}$ and $\varepsilon_{\mathrm{r}}^{\prime \prime}$ for TGS. 3 et $4:$ Consequent variations of the parameters $v$ and $\alpha$ for the cavity. Comparison between calculated and experimental values.]

Les mesures ont été effectuées en utilisant la technique de la cavité double [6], qui permet de réaliser automatiquement la correction de dilatation de la cavité.

A partir des valeurs de $\varepsilon_{\mathrm{r}}^{\prime}, \varepsilon_{\mathrm{r}}^{\prime \prime}$ et des dimensions géométriques, la résolution numérique, en valeurs complexes, de l'équation caractéristique (3) donne les valeurs des paramètres $v$ et $\alpha$ de la résonance, que l'on peut confronter à l'expérience. Les graphiques 3 et 4 de la figure 8 présentent les résultats obtenus. Les courbes en traits pleins représentent les résultats du calcul et les petits cercles, les résultats expérimentaux.

On peut distinguer trois domaines de températures correspondant à des comportements différents de l'échantillon.

Entre la température ambiante et $44^{\circ} \mathrm{C}$ l'échantillon 
présente un comportement diélectrique normal. La fréquence de résonance $v$ varie en sens inverse du paramètre $\varepsilon_{\mathrm{r}}^{\prime}$, et l'amortissement $\alpha$ varie dans le même sens que le paramètre $\varepsilon_{\mathrm{r}}^{\prime \prime}$. Entre $44^{\circ} \mathrm{C}$ et $64^{\circ} \mathrm{C}$, domaine de températures où $\operatorname{tg} \delta>0,4$, l'échantillon présente un comportement métallique, qui se traduit surtout par une remontée de la fréquence de résonance. Mais on note aussi un tassement des variations de l'amortissement. Dans cette région l'accord entre les prévisions et les mesures est moins bon. En particulier l'amplitude de la remontée en fréquence observée est inférieure de $20 \%$ à la valeur calculée. Enfin au-delà de $64^{\circ} \mathrm{C}$, l'échantillon retrouve un comportement diélectrique normal, avec un bon accord entre les paramètres observés et les paramètres calculés.

5. Conclusion. - Une cavité hyperfréquence contenant un échantillon diélectrique peut être décrite, tout au moins dans les configurations géométriques simples, par une équation caractéristique. Lorsque la permittivité de l'échantillon est complexe, il faut utiliser aussi une fréquence complexe, dont la partie réelle représente la fréquence de résonance de la cavité, et la partie imaginaire caractérise l'amortissement de la résonance. Cette équation caractéristique décrit le comportement de la cavité quelle que soit la permittivité de l'échantillon. Nos investigations dans le domaine des permittivités élevées, associées à des pertes diélectriques importantes nous ont permis de prévoir deux types de comportements de l'échantillon : un comportement diélectrique normal où l'échantillon absorbe les champs électromagnétiques, et un comportement métallique où l'échantillon repousse les champs électromagnétiques. La transition entre ces deux comportements se produit dans un domaine étroit de valeurs de $\operatorname{tg} \delta$. Il est facile d'observer expérimentalement une excursion dans le domaine des comportements métalliques en étudiant en fonction de la température, une cavité contenant un échantillon monocristallin, ferroélectrique, de T. G. S., et résonnant sur un mode de la famille $\mathrm{TM}_{1 \mathrm{mo}}$. L'accord numérique entre les valeurs calculées et les valeurs mesurées est bon.

Nous pouvons donc conclure que l'équation caractéristique, et son interprétation complexe ont un domaine de validité très étendu, et peuvent être utilisés pour décrire toutes les résonances observables.

Leur utilisation pour la détermination des permittivités complexes permet d'envisager des cas qui ne seraient pas adaptés à un traitement par la théorie des perturbations.

\section{Bibliographie}

[1] Poinsot, A. et Joly, J. C., Onde Electrique, 52 (1972) 223.

[2] Boudouris, G., Cavités électromagnétiques (Dunod, Paris) 1971, p. 65.

[3] EugÈne, C. E., IEEE Trans. MTT 21 (1973) 847.

[4] Hill, R. M. et IChiki. S. K., Phys. Rev. 128 (1962) 1140.
[5] Pereverzeva, L. P. et Poplavko, Y. M., Sov. Phys. Crystallogr. 18 (1974) 492.

[6] Poinsot, A., Sliwa, S. et Vosgien, G., Rev. Phys. Appl. (à paraître).

\section{ANNEXE}

\section{Méthode numérique utilisée pour la résolution des équations caractéristiques}

1. MÉTHOde DE DICHOTOMIE POUR LA RÉSOLUTION $f(x)=0$ DANS LE PLAN RÉEL. - Nous nous bornerons à rappeler brièvement cette méthode très efficace pour la résolution dans le plan réel d'une équation quelconque de la forme $f(x)=0$.

Elle permet d'obtenir toutes les solutions situées dans un intervalle donné $\left[x_{1}, x_{2}\right]$ que l'on explore par pas successifs de valeur $p$, en comparant à chaque pas les signes de $f\left(x_{1}+n p\right)$ et $f\left[x_{1}+(n-1) p\right]$.

Deux signes différents indiquent la présence d'une solution dans l'intervalle

$$
\left[a=x_{1}+(n-1) p, \quad b=x_{1}+n p\right] \text {. }
$$

On calcule alors le milieu $m=\frac{a+b}{2}$ de l'intervalle et $f(m)$. L'examen des signes de $f(a)$ et $f(m)$ permet de déterminer lequel des deux intervalles $[a, m]$ ou $[m, b]$ contient la solution. On réitère alors le processus de bissection sur l'intervalle contenant la solution jus- qu'à ce que celle-ci se trouve localisée dans un intervalle de largeur inférieure ou égale à la précision demandée.

Dès qu'une solution a été ainsi calculée, on reprend l'exploration de l'intervalle $\left[x_{1}, x_{2}\right]$ au point où on l'avait laissée. Si le pas d'exploration est trop grand il est possible que des solutions échappent, par groupes de deux, à la recherche. En pratique quelques essais suffisent pour déterminer une valeur adéquate du pas. Signalons cependant que les solutions des équations caractéristiques étudiées ici ne sont pas toujours régulièrement espacées. Il arrive de trouver deux solutions très proches l'une de l'autre, ceci provenant de la forme en marche d'escaliers des courbes caractéristiques.

2. RÉSOLUTION D'UNE ÉQUATION CARACTÉRISTIQUE DANS LE PLAN COMPLEXE. - Remarquons d'abord que cette résolution nécessite le calcul de fonctions de Bessel d'argument complexe. Nous utilisons pour cela 
un programme très simplifié qui repose uniquement sur l'expression de ces fonctions sous forme d'une série. Nous avons vérifié que notre programme donne sur l'axe réel et sur l'axe imaginaire les valeurs tabulées des fonctions $J_{l}, Y_{l}, K_{l}$ et $I_{l}$. En dehors des axes nous avons testé notre programme par rapport à des programmes plus importants. Nous avons toujours trouvé un accord excellent pour toutes les valeurs de l'argument susceptibles d'intervenir dans nos études.

Pour la résolution de l'équation caractéristique dans le plan complexe, tous les paramètres physiques sont fixés sauf $v$ et $\alpha$ (notre programme permet aussi une résolution par rapport à $\varepsilon^{\prime}$ et $\varepsilon^{\prime \prime}$ lorsque $v$ et $\alpha$ sont fixés). Il faut alors résoudre un système de deux équations (non linéaires) à deux inconnues du type :

$$
\left\{\begin{array}{l}
f(v, \alpha)=0 \\
g(v, \alpha)=0
\end{array}\right.
$$

où $f$ et $g$ représentent respectivement la partie réelle et la partie imaginaire de l'équation caractéristique.

La résolution consiste à trouver toutes les solutions situées à l'intérieur d'un rectangle $v \in\left[v_{1}, v_{2}\right]$, $\alpha \in\left[\alpha_{1}, \alpha_{2}\right]$. Dans un premier temps nous faisons une résolution graphique en traçant point par point les courbes représentatives des équations (1) dans le plan $(v, \alpha)$. Pour cela on calcule pour une valeur $\alpha_{0}$ fixée les solutions de

$$
\left\{\begin{array}{l}
f\left(v, \alpha_{0}\right)=0 \\
g\left(v, \alpha_{0}\right)=0 .
\end{array}\right.
$$

Ces deux équations sont indépendantes et chacune d'elles ne comporte qu'une inconnue. La méthode de bissection donne toutes les solutions appartenant à l'intervalle $\left[v_{1}, v_{2}\right]$. Pour décrire tout le rectangle de recherche on procède à un balayage par pas successifs sur la valeur de $\alpha_{0}$ depuis $\alpha_{1}$ jusqu'à $\alpha_{2}$. L'expérience nous a montré qu'un balayage logarithmique portant sur 3 ou 4 décades était le plus souvent nécessaire. La figure 1 montre un exemple de graphique obtenu à l'issue de cette phase de résolution. Les solutions de l'équation caractéristique se trouvent aux intersections des courbes.

Cette résolution graphique permet une première localisation des solutions. On pourrait d'ailleurs atteindre une bonne précision par agrandissements successifs, mais la méthode est long'ue. Aussi avons-nous mis au point un programme de calcul précis des solutions qui constitue la seconde phase de la résolution.

Ce programme opère sur un rectangle du plan $(v, \alpha)$ plus petit que le rectangle initial, et tel que chacune des équations (2) ait une solution et une seule à l'intérieur du rectangle. Le but de la phase graphique de résolution e j $^{\text {p }}$ écisément la détermination d'un tel rectangle à l'intérieur duquel il est possible de définir une fonction

$$
h\left(\alpha_{0}\right)=v_{1}\left(\alpha_{0}\right)-v_{2}\left(\alpha_{0}\right)
$$

où $v_{1}\left(\alpha_{0}\right)$ est la solution de $f\left(v_{1}, \alpha_{0}\right)=0$ et $v_{2}\left(\alpha_{0}\right)$ la solution de $g\left(v_{2}, \alpha_{0}\right)=0$. Résoudre l'équation carac-

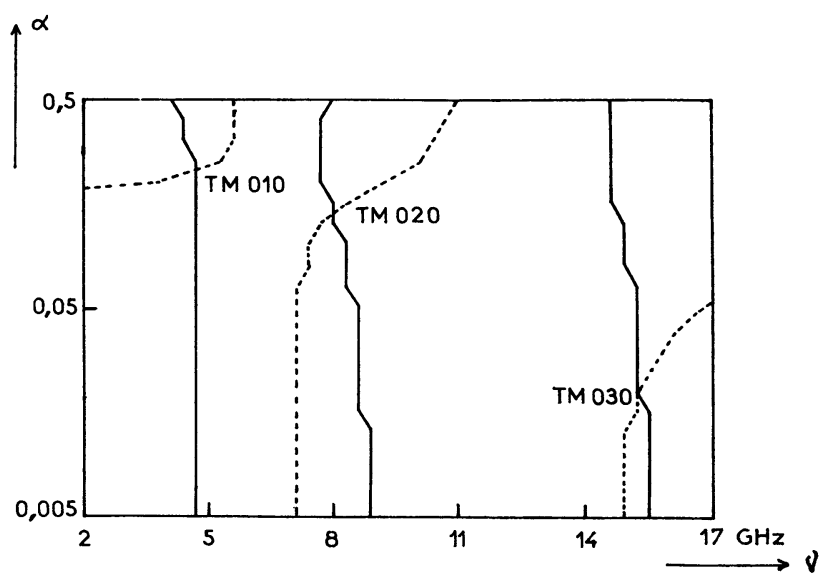

FIG. 1. - Exemple de graphique obtenu à l'issue de la $1^{\text {re }}$ phase du calcul. Les paramètres physiques sont : $\varepsilon_{\mathrm{r}}^{\prime}=32, \varepsilon_{\mathrm{r}}^{\prime \prime}=32$, $R_{1}=1 \mathrm{~mm}, R_{2}=19,98 \mathrm{~mm}$, modes $\mathrm{TM}_{\text {omo }}$. La seconde phase du calcul permet de préciser les solutions :

$$
\begin{aligned}
& \text { Mode } \mathrm{TM}_{010}\left\{\begin{array}{l}
v=4630,5 \mathrm{MHz} \\
\alpha=0,217 \\
\text { Mode } \mathrm{TM}_{020}
\end{array}\right. \\
& \text { Mode } \mathrm{TM}_{030} \quad \begin{array}{l}
v=28,8 \mathrm{MHz} \\
\alpha=0,140
\end{array} \\
& \text { non calculé. }
\end{aligned}
$$

[Example of a graphic obtained in the first phase of calculus. Physical parameters are : $\varepsilon_{\mathrm{r}}^{\prime}=32, \varepsilon_{\mathrm{r}}^{\prime \prime}=32, R_{1}=1 \mathrm{~mm}$, $R_{2}=19.98 \mathrm{~mm}$, modes $\mathrm{TM}_{\mathrm{omo}}$. The second phase of calculus gives more accurate solutions :

$$
\begin{aligned}
& \text { Mode } \mathrm{TM}_{010}\left\{\begin{array}{l}
v=4630.5 \mathrm{MHz} \\
\alpha=0.217
\end{array}\right. \\
& \text { Mode } \mathrm{TM}_{020}\left\{\begin{array}{l}
v=8020.8 \mathrm{MHz} \\
\alpha=0.140
\end{array}\right. \\
& \text { Mode } \mathrm{TM}_{030} \text { non calculated.] }
\end{aligned}
$$

téristique revient alors à résoudre $h\left(\alpha_{0}\right)=0$ ce qui peut se faire efficacement par la méthode de dichotomie. La figure 2 illustre la façon de déterminer un rectangle propice à la phase de résolution numérique.

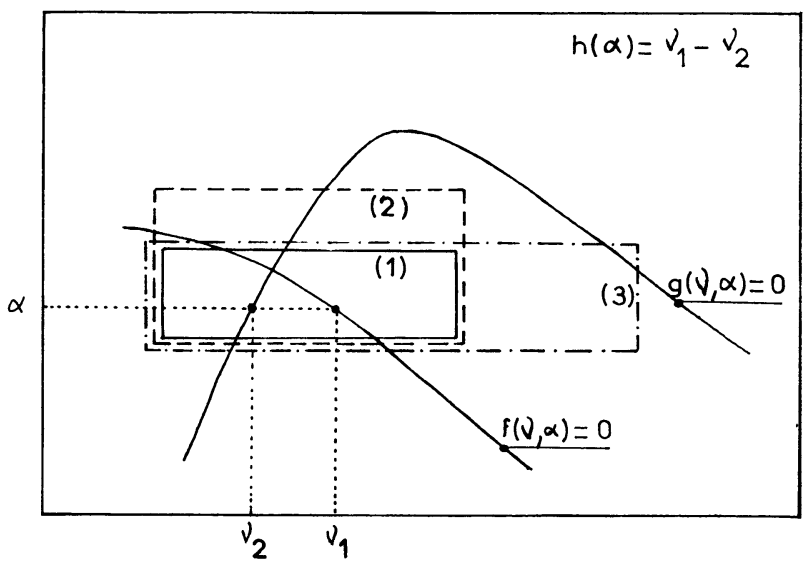

Fig. 2. - Choix d'un rectangle dans le plan $(v, \alpha)$ pour la phase numérique de la résolution. Le rectangle (1) convient, $h(\alpha) y$ est défini en tout point. Le rectangle (2) ne convient pas, $h(\alpha)$ n'est pas défini en tout point. Le rectangle (3) ne convient pas, la définition de $h(\alpha)$ n'est pas unique en tout point.

[Choice of rectangle in the $(v-\alpha)$ plane for the numerical phase of resolution : rectangle (1) is a good one, $h(\alpha)$ is defined at any point ; rectangle (2) is not good, $h(\alpha)$ is not defined at any point ; rectangle (3) is not good, $h(\alpha)$ is not univalued at any point.] 
Cette méthode de résolution, calquée sur un processus utilisable pour une résolution manuelle, est assez lourde mais ses avantages sont importants. Les programmes sont simples et découlent entièrement d'une bonne maitrise d'un programme de dichotomie. Les solutions sont localisées et identifiées dès la première phase graphique. Il n'est donc pas question que des solutions échappent au calcul et on peut calculer toutes les solutions que l'on désire et seulement celles-là. 